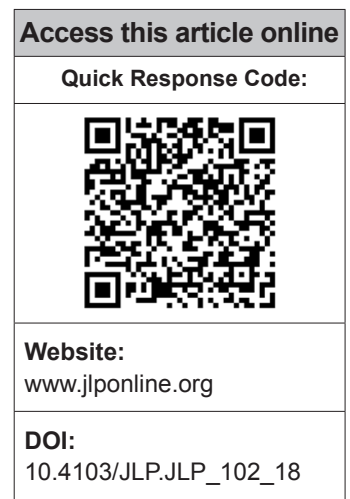

Department of Laboratory Medicine, All India Institute of Medical Sciences,

${ }^{1}$ Department of Laboratory Medicine, Primus Super Speciality Hospital, New Delhi, India

Address for correspondence:

Dr. Asok Kumar Mukhopadhyay, Department of Laboratory Medicine, All India Institute of Medical Sciences, New Delhi - 110 029, India. E-mail:mukhoak1953@ gmail.com

Submission: 28-07-2018 Accepted: 26-11-2018

\title{
Volume, conductivity, and scatter parameters of leukocytes as early markers of sepsis and treatment response
}

\author{
Parul Arora, Praveen Kumar Gupta', Raghavendra Lingaiah, \\ Asok Kumar Mukhopadhyay
}

\begin{abstract}
:
INTRODUCTION: Morphologic changes in the size and granularity of leukocytes seen in sepsis could be measured using the volume, conductivity, and scatter (VCS parameters) from the automated hematology analyzers. The objective of this study is to find the clinical usefulness of VCS parameters as possible indicators of sepsis and to determine the effect of treatment on these parameters.
\end{abstract}

METHODS: This observational study was conducted in a tertiary level hospital in India. Hemogram and VCS parameters obtained from LH 750 (Beckman coulter, Fullerton, CA) from 134 proven blood culture-positive cases of sepsis were reviewed on the day of culture positivity (day 0 ), day 3 , and day 7 were analyzed and compared with those of samples from otherwise healthy 100 participants. Statistical analysis of data was done, and cutoff value was established using receiver-operator characteristic curve.

RESULTS: Out of 134 culture-positive cases, 55.2\% $(n=74)$ Gram-negative and $44.8 \%(n=60)$ Gram-positive bacteria were isolated. The mean neutrophil volume (MNV) and mean monocyte volume (MMV) were higher in the sepsis group compared to that of the control group (165.43 $\pm 18.21 \mathrm{vs}$. $140.59 \pm 7.6, P=0.001$ for MNV and $179.8 \pm 14.16$ vs. $164.54 \pm 9.6, P=0.001$ for MMV). A significant decrease in MNV and MMV was observed with the initiation of the treatment. Significant changes in scatter and conductivity parameters were also noticed. A cutoff value of 150.2 for MNV gave a sensitivity and specificity of $79.1 \%$ and $95 \%$, respectively, with an area under the curve (AUC) of $92.3 \%$. With a cutoff of 168.3 , MMV had a sensitivity of $80.6 \%$ and specificity of $77.5 \%$, AUC of $83 \%$.

CONCLUSION: VCS parameters such as MNV and MMV can be easily obtained by an automated hematology analyzer and could be used for early detection and therapeutic response in sepsis.

Key words:

Automated analyzer, monocyte, neutrophil, sepsis, volume, conductivity, scatter parameters

\section{Introduction}

Cepsis is a major cause of morbidity Sand mortality in hospitalized patients worldwide, especially in a developing country like India. The early diagnosis of sepsis is extremely important to reduce the high mortality by prompt initiation of antibiotics. Blood culture remains the

This is an open access journal, and articles are distributed under the terms of the Creative Commons Attribution-NonCommercial-ShareAlike 4.0 License, which allows others to remix, tweak, and build upon the work non-commercially, as long as appropriate credit is given and the new creations are licensed under the identical terms.

For reprints contact: reprints@medknow.com gold standard for diagnosing bacteremia or sepsis. ${ }^{[1]}$ Although viral and fungal agents are also implicated in sepsis, bacteria is still the most important implicated agent. Complete hemogram along with differential leukocyte count are the first parameters to suggest infections. ${ }^{[2]}$ Increased total leukocyte count (TLC), immature/ total neutrophil ratio, and neutrophilia are associated findings in sepsis. The characteristic morphological changes in the

How to cite this article: Arora P, Gupta PK, Lingaiah R, Mukhopadhyay AK. Volume, conductivity, and scatter parameters of leukocytes as early markers of sepsis and treatment response. J Lab Physicians 2019;11:29-33. 
size of the cell, the density of the nucleus, nuclear lobes, along with the presence of toxic granules, vacuolization, and occasional Döhle bodies, are characteristically seen in sepsis. ${ }^{[3,4]}$ The identification of these characteristics on peripheral smear is a subjective finding, the identification of which depends on the expertise in microscopy. Blood culture too has limitations, such as it has low-sensitivity, takes time for growth, and is easily amenable to contamination. Moreover, a positive blood culture report takes at least $48 \mathrm{~h}$, which may delay the initiation of early antibiotic therapy by the treating physician. ${ }^{[1]}$

The newer hematology analyzers have completely revolutionized the hemogram analysis. The Coulter $\mathrm{LH}$ 750 hematology analyzer (Beckman Coulter, Fullerton, CA, USA) obtains data from $>8000$ white blood cells (WBCs) in a few seconds, using impedance to measure the cell volume $(\mathrm{V})$ for the size of all cells, radio-frequency opacity to characterize the conductivity $(\mathrm{C})$ for the internal composition of each cell, and a laser beam to measure the light scatter (S) for cytoplasmic granularity and nuclear structure. This volume, conductivity, and scatter (VCS) technology, thus helps in quantifying not only the number of cells but also the internal characteristics of a cell which can be compared with the morphological appearance of WBCs on peripheral smear examination. ${ }^{[5-7]}$

This VCS feature can be used as a tool for the early diagnosis of sepsis. ${ }^{[7-10]}$ The usefulness of VCS parameters can be assessed by their changes at the time of sepsis. Although there have been previous studies which support the same, their clinical relevance can be judged by the change in parameters with the course of antibiotic treatment..$^{[9,11,12]}$ Therefore, in the present study, we have tried to investigate the significance of VCS parameters such as mean neutrophil volume (MNV), conductivity (MNC), and scatter (MNS), mean monocyte volume (MMV), conductivity (MMC), and scatter (MMS) in diagnosing bacteremia and correlate them to antibiotic course. We have also tried to find a significant cutoff value of MNV and MMV that could aid to be supportive evidence in diagnosing bacteremia or sepsis.

\section{Methods}

This retrospective observational study was conducted at a tertiary care hospital in North India over a period of 6 months.

\section{Data collection}

Hemogram and VCS parameters of 134 proven blood culture-positive cases of sepsis were reviewed on the day of culture positivity (day 0), day 3, and day 7 were analyzed. Samples were processed in hematology analyzer LH 750 (Beckman Coulter, Fullerton, CA, USA) within $4 \mathrm{~h}$ after receiving in the laboratory. The patient's medical records were also reviewed for clinical correlation. Patients with any known hematological disorder or malignancy, patients on multivitamins/hematinics, and patients with burns/trauma or those with macrocytosis (MCV >110 FL) on day 0 were excluded from the study.

\section{Controls}

Hemogram and VCS parameters from the samples of 100 healthy participants from blood bank of this hospital were taken as control. These parameters were reviewed and compared with that of the cases. Control showed no symptoms of infection, such as fever, and had WBC and differential counts within the normal limits.

\section{Statistical analysis}

The data were collected and compiled in Excel, following which statistical analysis was done using the IBM SPSS Statistics (SPSS for Windows, Version 16.0. Chicago, SPSS Inc). Comparison of means between the two groups was examined by using $t$-test where the data had a normal distribution, and by Wilcoxon Rank-Sum test where the data had nonnormal distributions. For comparison of more than two groups, ANOVA was used for normal distributions and the Kruskal-Wallis test for nonnormal distributions. A value of $P<0.05$ was considered as statistically significant. Receiver operating characteristic (ROC) curve analysis was used to determine the power of variables to calculate the significant cutoff levels to determine the sensitivity, specificity, and area under the ROC curve.

\section{Results}

Data from 134 patients with a positive bacterial blood culture and 100 controls were analyzed.

\section{Baseline characteristics}

The baseline characteristics of cases and controls are given in Table 1. Mean age and male:female ratio were similar between the two groups $(P>0.05)$. Out of the 134 bacterial culture-positive cases, $55.2 \%(n=74)$ were Gram negative and $44.8 \%(n=60)$ were Gram positive. Staphylococcus aureus $(32 \%, n=43)$ was the most common organism isolated followed by Escherichia coli (17.2\%, $n=23)$.

TLC, the percentage of neutrophils (NE \%), and percentage of monocytes $(\mathrm{MO} \%)$ were evaluated in the cases and controls along with their VCS parameters. It was observed that the TLC and NE\% were significantly higher in cases as compared to that of the controls $(P=0.001)$. However, the $\mathrm{MO} \%$ was not significantly increased $(P=0.74)$.

\section{Volume, conductivity, and scatter parameters Neutrophils \\ A significantly higher MNV was seen in sepsis patients}


Table 1: Baseline characteristics and volume, conductivity, and scatter parameters of the study population

\begin{tabular}{lccc}
\hline & Controls $(n=100)$ & Cases $(n=134)$ & $P$ \\
\hline Mean age (years) & $32.9 \pm 8.3$ & $32.2 \pm 10.6$ & 0.7 \\
Male:female & $19: 21$ & $69: 65$ & - \\
MCV (fL) & $92 \pm 6.7$ & $90 \pm 9.9$ & 0.38 \\
\hline TLC $\left(\times 10^{3} / \mu \mathrm{L}\right)$ & $7.8(4-9.5)^{*}$ & $11.3(2-51)^{*}$ & 0.001 \\
Neutrophils $(\%)$ & $56.2 \pm 13.24$ & $72 \pm 17.29$ & 0.001 \\
MNV & $140.59 \pm 7.6$ & $165.43 \pm 18.21$ & 0.001 \\
MNC & $155.36 \pm 4.27$ & $128.9 \pm 7.8$ & 0.001 \\
MNS & $142.26 \pm 6.7$ & $138.58 \pm 9.7$ & 0.027 \\
Monocytes (\%) & $7.8(4.3-10.6)^{*}$ & $7.6(0.4-45.1)^{*}$ & 0.74 \\
MMV & $164.54 \pm 9.6$ & $179.8 \pm 14.16$ & 0.001 \\
MMC & $130.6 \pm 2.9$ & $110.8 \pm 6.3$ & 0.001 \\
MMS & $86.4 \pm 2.5$ & $91.16 \pm 5.3$ & 0.001 \\
\hline
\end{tabular}

Values are expressed as mean \pm SD for parametric or median* (minimum-maximum) for nonparametric results. SD = Standard deviation, MCV = Mean corpuscular volume, TLC = Total leukocyte count, MNV = Mean neutrophil volume, MNC = Mean neutrophil conductivity, MNS = Mean neutrophil scatter, $\mathrm{MMV}=$ Mean monocyte volume, $\mathrm{MMC}=$ Mean monocyte conductivity, and MMS = Mean monocyte scatter

than that of the controls $(165.43 \pm 18.21$ vs. $140.59 \pm 7.6$, $P=0.001)$. MNC (128.9 \pm 7.8 vs. $155.36 \pm 4.27, P=0.001)$, and MNS (138.58 \pm 9.7 vs. $142.26 \pm 6.7, P=0.027)$ were significantly lower in cases as compared to that of the controls.

\section{Monocytes}

Similarly, monocytes showed a significantly higher mean volume (MMV) in the sepsis patients than that of the controls $(179.8 \pm 14.16$ vs. $164.54 \pm 9.6, P=0.001)$. MMC $(110.8 \pm 6.3$ vs $130.6 \pm 2.9, P=0.001)$ was found to be lower in cases when compared with the controls.

Changes in white blood cell and volume, conductivity, and scatter parameters with treatment

In 134 blood culture-proven cases of sepsis, the changes in WBC and VCS parameters were evaluated on successive measurements on day 3 and day 7 after the antibiotic treatment was initiated [Table 2]. There was statistically significant decrease in MNV and MMV ( $P<0.001$ for both) values with antibiotic treatment, although NE\% $(P=0.72)$ and $\mathrm{MO} \%(P=0.33)$ did not decrease significantly. MNC, MMC, and MMS showed increased values with treatment which was statistically significant $(P<0.01)$.

Evaluation of sensitivity, specificity of volume, conductivity, and scatter parameters for predicting sepsis Sensitivity and specificity for TLC, percentage neutrophils, monocytes, MNV, and MMV were calculated at designated cutoff points that were taken from the ROC curves [Table 3 and Figure 1]. A cutoff value of 150.2 for MNV yielded a high sensitivity and specificity of $79.1 \%$ and $95 \%$, respectively, with an area under the curve (AUC) of $92.3 \%$. With a cutoff of 168.3 , MMV had a sensitivity of $80.6 \%$ and specificity of $77.5 \%$ with an AUC of $82.97 \%$.

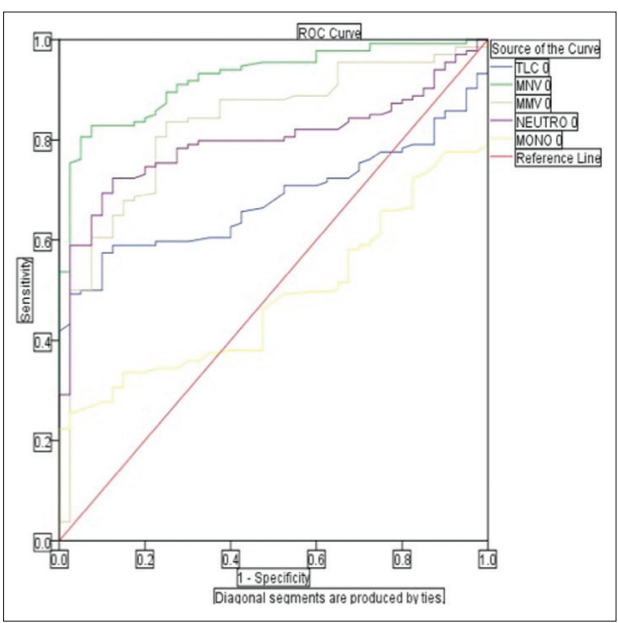

Figure 1: Receiver-operator characteristic curves for various neutrophil and monocyte parameters to differentiate the cases of sepsis from the healthy controls

\section{Discussion}

This retrospective observational study was conducted to evaluate the morphological changes of the WBCs obtained from the automated analyzer and its importance in predicting septicemia. LH 750 hematology analyzer examines the large numbers of cells to provide the reliable information about the number, size, nuclear complexity, and granularity of the WBCs. These parameters can be measured by the VCS technology. Such information has definitive implications in a clinical setting. ${ }^{[13,14]}$ In this study, we evaluated the VCS parameters of 134 septic patients with positive blood cultures and their response to treatment, comparing them to 100 healthy controls.

It is a known fact that septicemia is characterized by the appearance of immature neutrophils such as bilobed neutrophils, bands, metamyelocytes, and myelocytes in the peripheral blood. ${ }^{[3,4]}$ Morphologically, these immature neutrophils are larger and have a relatively simple nucleus. Such changes are reflected by raised MNV and decreased MNC and MNS in these cases compared to the controls. ${ }^{[1,12]}$ This study also reported such a change in pattern in monocytes which shows their role in response to the infections.

This study shows a retrospective analysis of the VCS parameters on the day of diagnosing septicemia and to find the effect of treatment, on day 3 and day 7. It was seen that the NE\% showed no statistical change [Table 2], $(P=0.7)$ with the treatment, which shows that it cannot be used to judge the response of treatment from the laboratory point of view. At the same time, it was observed that there was statistically significant decrease in size of the neutrophils and monocytes confirmed by the normalization of MNV and MMV [Table 2] ( $P<0.001$ for both). The finding 
Table 2: Changes in white blood cell and volume, conductivity, and scatter parameters with treatment $(n=134)$

\begin{tabular}{lccccccc}
\hline & \multicolumn{3}{c}{ Mean/median* value } & \multicolumn{3}{c}{$\boldsymbol{P}$} \\
\cline { 2 - 7 } & Day 0 & Day 3 & Day 7 & Overall & Day (0 vs. 3) & Day (0 vs. 7) & Day (3 vs. 7) \\
\hline Neutrophils (\%) & $75.31 \pm 15.35$ & $74.86 \pm 14.18$ & $74.15 \pm 16.63$ & 0.7 & 0.77 & 0.40 & 0.62 \\
MNV & $166.20 \pm 15.79$ & $158.20 \pm 15.49$ & $155.04 \pm 14.47$ & $<0.001$ & $<0.001$ & $<0.001$ & 0.001 \\
MNC & $127.57 \pm 7.51$ & $130.85 \pm 6.65$ & $129.38 \pm 7.14$ & $<0.001$ & $<0.01$ & 0.12 & 0.14 \\
MNS & $137.35 \pm 11.62$ & $139.70 \pm 7.35$ & $140.03 \pm 9.30$ & 0.03 & 0.06 & 0.03 & 0.70 \\
Monocytes (\%) & $7.8^{*}(0.4-33.6)$ & $7.3^{*}(0.6-25.2)$ & $6.8^{*}(0.4-28.8)$ & 0.33 & 0.50 & 0.03 & 0.10 \\
MMV & $182.01 \pm 15.58$ & $178.07 \pm 11.65$ & $176.28 \pm 10.63$ & $<0.001$ & 0.001 & $<0.001$ & 0.012 \\
MMC & $110.56 \pm 6.13$ & $111.57 \pm 7.39$ & $112.94 \pm 8.30$ & 0.003 & 0.12 & 0.002 & 0.04 \\
MMS & $90.18 \pm 5.22$ & $92.16 \pm 4.62$ & $93.09 \pm 3.73$ & $<0.001$ & $<0.001$ & $<0.001$
\end{tabular}

Values are expressed as mean \pm SD for parametric or median* (minimum-maximum) for non-parametric results. SD $=$ Standard deviation, MCV $=$ Mean corpuscular volume, TLC $=$ Total leukocyte count, MNV = Mean neutrophil volume, MNC = Mean neutrophil conductivity, MNS = Mean neutrophil scatter, MMV = Mean monocyte volume, $\mathrm{MMC}=$ Mean monocyte conductivity, MMS = Mean monocyte scatter

Table 3: Determination of cutoffs, sensitivity, specificity, and area under curve for mean neutrophil volume and mean monocyte volume

\begin{tabular}{llcccccc}
\hline & Differential groups & $\begin{array}{l}\text { Cutoff } \\
\text { points }\end{array}$ & Sensitivity & Specificity & Area under & \multicolumn{2}{c}{$95 \% \mathrm{Cl}$} \\
\cline { 7 - 8 } & & & & & curve (\%) & Lower bound & Upper bound \\
\hline Controls versus & TLC $\left(\times 10^{3} / \mu \mathrm{L}\right)$ & 10.05 & 57.5 & 87.5 & 68.1 & 60.5 & 75.6 \\
cases & Neutrophil $(\%)$ & 60.05 & 79.9 & 57.5 & 79.8 & 73.2 & 86.4 \\
& Monocyte $(\%)$ & 7.15 & 51.5 & 32.5 & 48.3 & 39.8 & 56.8 \\
& MNV & $\geq 150.2$ & 79.1 & 95 & 92.3 & 88.3 & 96.3 \\
& MMV & $\geq 168.3$ & 80.60 & 77.50 & 83.0 & 76.0 & 89.9 \\
\hline
\end{tabular}

$\mathrm{Cl}=$ Confidence interval, TLC = Total leukocyte count, $\mathrm{MNV}=$ Mean neutrophil volume, $\mathrm{MMV}=$ Mean monocyte volume

is further supported by a significant increase in MNC and MNS (though not significant), which reflects the normalization of WBCs in the peripheral blood causing immature neutrophils to be removed by apoptosis and being replaced by mature ones, thus increasing granularity and nuclear complexity with the treatment.

We further evaluated that whether a cutoff value can be determined which can be used in clinical practice to identify the cases of septicemia on the initial hemogram run on automated analyzers. ROC curve was computed to compare the sensitivity and specificity of the MNV and MMV. It was observed that taking a cutoff of $\geq 150.1, \mathrm{MNV}$ gave a sensitivity of $79.85 \%$ and a specificity of $95 \%$ with an AUC of 92.3 . The value is slightly different from studies from other parts of the world, which suggests differences in the study population and might reflect in their VCS parameters also. ${ }^{[7,11,12,14]}$ It is, therefore, important to establish normal ranges of these VCS parameters in one's own population before interpreting them in clinical significance.

Other investigators have also reported MNV as having higher sensitivity for predicting infection than TLC and the percentage of neutrophils. If the diagnosis of infection had been based only on the WBC count or the percentage of neutrophils, the correct diagnosis easily could have been overlooked. Therefore, we believe that the MNV has the potential to be used as an additional indicator of acute bacterial infection, particularly when the other hematologic parameters do not correlate well with the patient's clinical signs and symptoms. ${ }^{[7,8,13,15,16]}$

WBC counts seem to be an insensitive discriminator for the systemic infections ${ }^{[17,18]}$ because sepsis can be associated with normal or decreased leukocytes caused by an increased turnover and/or toxic suppression of bone marrow maturation. ${ }^{[12]}$ Clinical scores with regard to organ failure and outcome such as the Acute Physiology and Chronic Health score should also be used to quantitatively evaluate the patient group. Other markers that can be used in similar settings include C-reactive protein and procalcitonin. ${ }^{[19,20]}$ However, they are relatively expensive. A combination of markers can also be used to increase the diagnostic yield. ${ }^{[21,22]}$

Several other clinical applications of the VCS technology remain to be studied. With the vast amount of data that could be obtained relating to the morphologic features of neutrophils, lymphocytes, monocytes, and eosinophils separately, the prospects of their clinical use are promising. Other possible clinical applications could include the early diagnosis of other infections like viral infections that are known to change the morphologic features of lymphocytes. ${ }^{[23]}$

\section{Conclusion}

VCS parameters can be an important diagnostic tool that can serve as an adjunct to the clinical diagnosis of 
bacterial sepsis and can help in the early initiation of empirical antibiotic therapy.

\section{Acknowledgments}

We are thankful to the technical staff of this laboratory for their cooperation while conducting the study.

\section{Financial support and sponsorship}

Nil.

\section{Conflicts of interest}

There are no conflicts of interest.

\section{References}

1. Procop GW, Hartman JS, Sedor F. Laboratory tests in evaluation of acute febrile illness in pediatric emergency room patients. Am J Clin Pathol 1997;107:114-21.

2. Wile MJ, Homer LD, Gaehler S, Phillips S, Millan J. Manual differential cell counts help predict bacterial infection. A multivariate analysis. Am J Clin Pathol 2001;115:644-9.

3. Mathy KA, Koepke JA. The clinical usefulness of segmented vs. stab neutrophil criteria for differential leukocyte counts. Am J Clin Pathol 1974;61:947-58.

4. Wenz B, Gennis P, Canova C, Burns ER. The clinical utility of the leukocyte differential in emergency medicine. Am J Clin Pathol 1986;86:298-303.

5. Richardson-Jones A. An automated hematology instrument for comprehensive WBC, RBC, and platelet analysis. Am Clin Lab 1990;9:18-22.

6. Krause JR. Automated differentials in the hematology laboratory. Am J Clin Pathol 1990;93:S11-6.

7. Chaves F, Tierno B, Xu D. Quantitative determination of neutrophil VCS parameters by the coulter automated hematology analyzer: New and reliable indicators for acute bacterial infection. Am J Clin Pathol 2005;124:440-4.

8. Purohit AH, Kumar P, Sharma S, Kapil A, Gupta A, Mukhopadhyay AK. Volume, conductivity, and scatter parameters as diagnostic aid to bacterial sepsis: A tertiary care experience. Indian J Pathol Microbiol 2015;58:459-63.

9. Raimondi F, Ferrara T, Capasso L, Sellitto M, Landolfo F, Romano A, et al. Automated determination of neutrophil volume as screening test for late-onset sepsis in very low birth infants. Pediatr Infect Dis J 2010;29:288.

10. Suresh PK, Minal J, Rao PS, Ballal K, Sridevi HB, Padyana M, et al.
Volume conductivity and scatter parameters as an indicator of acute bacterial infections by the automated haematology analyser. J Clin Diagn Res 2016;10:EC01-3.

11. Lee AJ, Kim SG. Mean cell volumes of neutrophils and monocytes are promising markers of sepsis in elderly patients. Blood Res 2013;48:193-7.

12. Mardi D, Fwity B, Lobmann R, Ambrosch A. Mean cell volume of neutrophils and monocytes compared with C-reactive protein, interleukin- 6 and white blood cell count for prediction of sepsis and nonsystemic bacterial infections. Int J Lab Hematol 2010;32:410-8

13. Celik IH, Demirel G, Aksoy HT, Erdeve O, Tuncer E, Biyikli Z, et al. Automated determination of neutrophil VCS parameters in diagnosis and treatment efficacy of neonatal sepsis. Pediatr Res 2012;71:121-5.

14. Celik IH, Demirel G, Sukhachev D, Erdeve O, Dilmen U. Neutrophil volume, conductivity and scatter parameters with effective modeling of molecular activity statistical program gives better results in neonatal sepsis. Int J Lab Hematol 2013;35:82-7.

15. Abiramalatha T, Santhanam S, Mammen JJ, Rebekah G, Shabeer MP, Choudhury J, et al. Utility of neutrophil volume conductivity scatter (VCS) parameter changes as sepsis screen in neonates. J Perinatol 2016;36:733-8.

16. Park SH, Park CJ, Lee BR, Nam KS, Kim MJ, Han MY, et al. Sepsis affects most routine and cell population data (CPD) obtained using the sysmex XN-2000 blood cell analyzer: Neutrophil-related CPD NE-SFL and NE-WY provide useful information for detecting sepsis. Int J Lab Hematol 2015;37:190-8.

17. Seebach JD, Morant R, Rüegg R, Seifert B, Fehr J. The diagnostic value of the neutrophil left shift in predicting inflammatory and infectious disease. Am J Clin Pathol 1997;107:582-91.

18. Pettilä V, Hynninen M, Takkunen O, Kuusela P, Valtonen M. Predictive value of procalcitonin and interleukin 6 in critically ill patients with suspected sepsis. Intensive Care Med 2002;28:1220-5.

19. Chan YL, Tseng CP, Tsay PK, Chang SS, Chiu TF, Chen JC. Procalcitonin as a marker of bacterial infection in the emergency department: An observational study. Crit Care 2004;8:R12-20.

20. Wang S, Chen D. The correlation between procalcitonin, C-reactive protein and severity scores in patients with sepsis and their value in assessment of prognosis. Zhonghua Wei Zhong Bing Ji Jiu Yi Xue 2015;27:97-101.

21. Faix JD. Biomarkers of sepsis. Crit Rev Clin Lab Sci 2013;50:23-36.

22. Bloos F. Clinical diagnosis of sepsis and the combined use of biomarkers and culture- and non-culture-based assays. Methods Mol Biol 2015;1237:247-60.

23. Zhu Y, Cao X, Tao G, Xie W, Hu Z, Xu D, et al. The lymph index: A potential hematological parameter for viral infection. Int $J$ Infect Dis 2013;17:e490-3. 